\title{
A Case with Atypical Clinical Course Diagnosed as Osteoarthritis, Osteonecrosis, Subchondral Insufficiency Fracture, or Rapidly Destructive Coxopathy
}

\author{
Yukio Nakamura $^{*},{ }^{1}$, Mikio Kamimura ${ }^{2}$, Keijiro Mukaiyama ${ }^{1}$, Shota Ikegami ${ }^{1}$, Shigeharu Uchiyama ${ }^{1}$ \\ and Hiroyuki Kato ${ }^{1}$ \\ ${ }^{1}$ Department of Orthopaedic Surgery, Shinshu University School of Medicine, Asahi 3-1-1, Matsumoto 390-8621, Japan \\ ${ }^{2}$ Center of Osteoporosis and Spinal Disorders: Kamimura Orthopaedic Clinic, Matsumoto 399-0021, Japan
}

\begin{abstract}
Osteonecrosis (ON), subchondral insufficiency fracture (SIF), and rapidly destructive coxopathy (RDC) are considered to be clinically different disorders despite exhibiting several overlapping features. We encountered an elderly female patient with an atypical clinical course who was radiographically diagnosed as having osteoarthritis (OA), ON, SIF, and/or RDC over a long-term follow-up. In this case, radiographic diagnosis was apparently affected by the timing of imaging evaluation and was challenging because of radiographic overlap and atypical disease progression. The disorders of OA, SIF, ON, and RDC might share a similar pathophysiology.
\end{abstract}

Keywords: OA, ON, RDC, SIF.

\section{INTRODUCTION}

The major and most frequent hip disorder is osteoarthritis (OA) [1]. Idiopathic and steroid- or alcohol-induced osteonecrosis $(\mathrm{ON})$ is also a disorder requiring urgent clarification of its pathophysiology [2]. Recently, subchondral insufficiency fracture (SIF) of the femoral head is a new concept in hip joint disorders [3]. Yet another hip condition in the elderly is rapidly destructive coxopathy (RDC) [4].

The pathophysiologies of the above disorders are considered to be distinct from one another [5-8]. It is generally believed that cartilage degeneration caused by repetitive mechanical stress is the primary pathophysiology of OA [9]. On the other hand, it has been reported that the major cause of ON is trauma that mechanically disrupts bone-supplying arteries [10]. SIF is considered to be caused by femoral head fracture [3]. Although various theories have been proposed for RDC pathophysiology, fracture has recently been implicated as a main cause [8]. ON and SIF share several similarities in terms of imaging findings [11]. The characteristic imaging feature of RDC is rapid femoral head destruction [8], and ON and SIF resemble RDC at the late to end stages of these conditions [11].

This report describes a patient who was initially diagnosed as having radiographic $\mathrm{OA}$ and then exhibited imaging features of ON and SIF, which finally appeared as radiographic RDC over a long-term course of follow-up.

\footnotetext{
*Address correspondence to this author at the Department of Orthopaedic Surgery, Shinshu University School of Medicine, Asahi 3-1-1, Matsumoto 390-8621, Japan; Tel: +81-263-37-2659; Fax: +81-263-35-8844;

E-mail: yxn14@aol.jp
}

\section{CASE REPORT}

A 90-year-old woman presented at our institution suffering from right hip joint pain that had been absent since an earlier visit when she was 83 years old. Her height was $153 \mathrm{~cm}$ and her body weight was $58 \mathrm{~kg}$. She had a history of hypertension. The clinical course of her joint pain was as follows: she initially complained of continued right hip joint pain at the age of 83 years and 3 months. Therefore, nonsteroidal anti-inflammatory drug (NSAID) administration and bisphosphonate (BP) treatment for osteoporosis were given. A mild thoracic vertebral compression fracture was also observed approximately 7 years earlier at the patient's first visit. Osteoporotic treatment with BP had been continued since that time. Right hip bone mineral density $(B M D) 7$ years prior and at present were $0.915(\mathrm{~T}$ score $=$ 1.7) and 1.094 (T score $=-0.1)$, respectively. Left hip BMD had risen from $0.702(\mathrm{~T}$ score $=-1.9) 7$ years earlier to 0.755 (T score $=-1.5)$ at present. During follow-up, she sometimes complained of mild hip joint pain. At the time of this report, her joint pain was substantially decreased.

Right hip range of motion (ROM) was slightly restricted at the patient's first visit, but did not worsen over 7 years.

A right hip plain radiograph at age 83 showed joint space narrowing (Kellgren-Lawrence [KL] grading II) [12] (Fig. 1a) that was diagnosed as radiographic OA. There was no acetabular dysplasia. At the age of 83 years and 8 months, plain radiographs revealed greater joint space narrowing, a band around the bone defect change-like osteonecrosis, and a concave shape to the right femoral head (Fig. 1b). The necrotic region of the femoral head had progressed a year after onset and was noticeably worsened at the age of 86 (Fig. 1c, d). Ensuing radiographs showed progressive destruction of the femoral head and osteosclerotic change of 


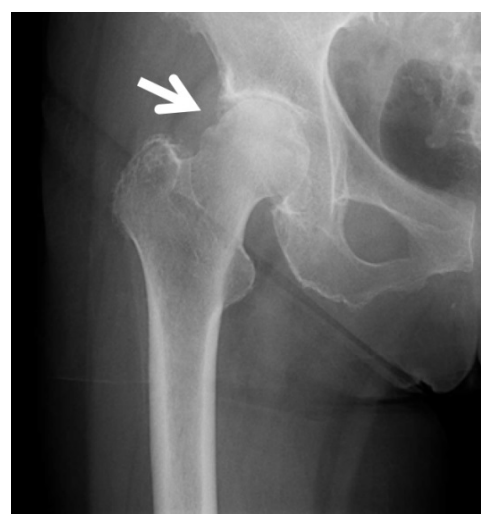

(a)

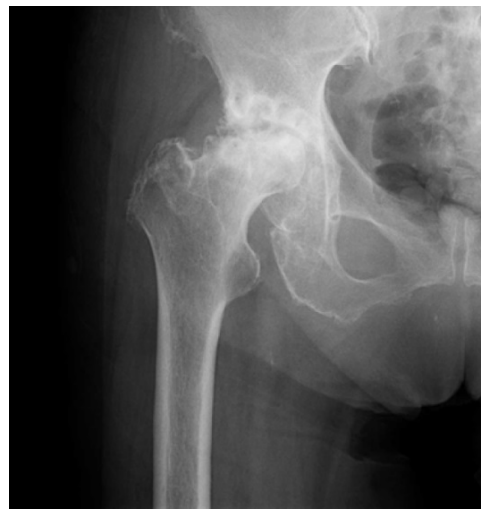

(d)

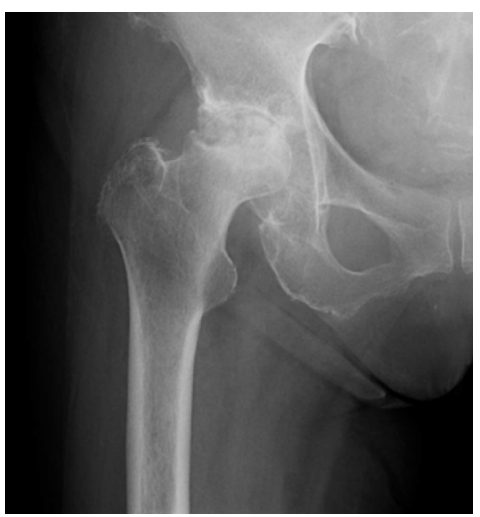

(b)

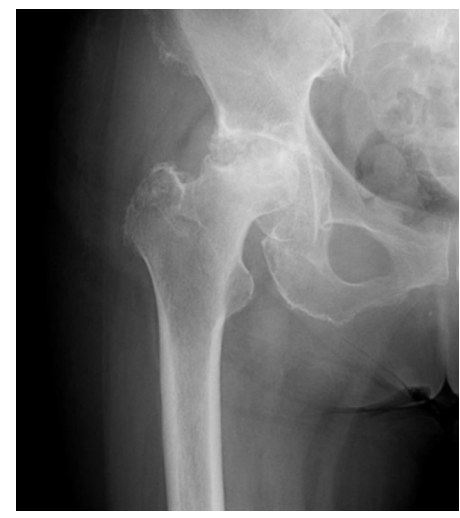

(c)

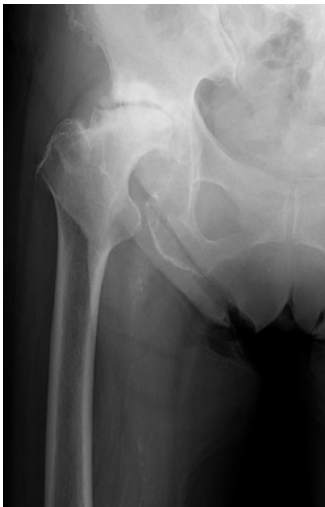

(e)

Fig. (1). (a) At the first visit, a plain radiograph of the right hip showed joint space narrowing (KL grading II). A laddering-shaped deformity in the right lateral proximal femoral head and fracture-like line were observed (white arrow). (b) Eight months after onset, a plain radiograph revealed increased joint space narrowing, a band around the necrotic area, and a concave shape to the right femoral head. (c) The necrotic region of the right femoral head became noticeably worse a year after onset. (d) The right femoral head had collapsed at 3 years after onset. (e) A recent radiograph showed progressive destruction of the right femoral head and the remaining joint line exhibited osteosclerotic change. Note that the patient did not report any joint pain.

the remaining joint line (Fig. 1e). In addition, radiographs retrospectively uncovered a laddering-shaped deformity in the right lateral proximal femoral head and a fracture-like line that were consistent with the necrotic region (Fig. 1a, arrow).

The particularity of this case was that 1) the patient was initially diagnosed as having radiographic OA, 2) joint pain was markedly improved after a few months, 3 ) the femoral head degenerated over several years in the absence of joint pain, and 4) radiographic diagnosis changed based on the timing of disease progression.

The patient was informed that the data from her case would be submitted for publication and gave her consent.

\section{DISCUSSION}

In the present case, plain radiographs revealed joint space narrowing that was considered to reflect early-stage OA [13]. However, at the age of 83 years and 3 months old, the patient's joint pain had disappeared. Radiographs showed a partial collapse of the femoral head, which was then considered to be ON. However, ON was ruled out as she exhibited progressive radiographic joint space narrowing.
The shape of the femoral head collapse was different from that in SIF. Finally, radiographs uncovered progressive destruction of the femoral head, which was considered to be due to RDC. Therefore, it appears that radiographic diagnosis varies depending on the timing of imaging evaluation.

$\mathrm{ON}$ is considered to be a disorder with joint destruction and collapse caused by an ischemic femoral head. ON of the femoral head is classified into idiopathic and secondary forms [14]. The revised criteria for idiopathic ON diagnosis were established in June 2001. They include 1) collapse of the femoral head (including crescent sign) without joint space narrowing or acetabular abnormality on radiograph images, 2) demarcating sclerosis in the femoral head without joint space narrowing or acetabular abnormality, 3) "cold in hot" on bone scans, 4) a low-intensity band on T1-weighted (T1W) MRI (band-like pattern), and 5) histological findings of trabecular and marrow necrosis. Idiopathic ON of the femoral head may be diagnosed if the patient fulfills two of these five criteria and does not exhibit bone tumor or dysplasia [6].

This case was initially considered to be of $\mathrm{ON}$ based on the condition of femoral head collapse $[6,11]$. However, the occurrence of radiographic $\mathrm{OA}$ immediately after the 
outbreak of the disorder and the patient's progressive joint line destruction were inconsistent with ON $[6,11]$.

Recently, SIF of the femoral head has been proposed as a new concept in femoral head collapse [3, 4]. The diagnosis of SIF has been summarized by Iwasaki et al. as follows: 1) radiographs appear normal or show collapse of the femoral head, joint space narrowing, and/or a linear patchy sclerotic area in the superior portion of the femoral head, 2) a bone marrow edema pattern is seen in the femoral head and/or neck on MRI, and 3) a subchondral low-signal intensity band on T1W MRI is evident, which is either serpiginous or parallel with the articular surface [7].

SIF is due to bone fragility usually secondary to osteoporosis or osteopenia and leads to subchondral fractures in the femoral head $[3,4]$. In contrast, $\mathrm{ON}$ is a condition of the vascular supply to the femoral head, resulting in articular surface collapse and eventual OA $[6,11,15]$. Ikemura et al. have reported that an irregular, serpiginous, convex shape to the articular surface and often discontinuous shape of a lowintensity band on T1W MRI suggest SIF. In contrast, a concave shape to the articular surface of the femoral head suggests ON $[6,11]$.

As our patient demonstrated joint space narrowing with femoral head destruction and a laddering-shaped deformity in the right lateral proximal femoral head on plain radiographs at onset, the primary clinical diagnosis was SIF. However, a concave shape to the articular surface was observed, which was counter-indicative of this condition.

$\mathrm{ON}$ and SIF share several common imaging features [6, $7,11]$. One of the characteristic features of RDC is rapid femoral head destruction [8], which can resemble findings of both ON and SIF at the late to end stages of these diseases [11]. In addition to rapid joint destruction 6-12 months from the onset of symptoms) RDC is characterized by disappearance of the joint space [8]. However, the details regarding its etiology and epidemiology have not yet been clarified. Several reports have suggested increased pelvic posterior inclination [15], aseptic necrosis of the femoral head [16], anti-inflammatory drug-induced joint destruction (analgesic hip) [17], and articular chondrocalcinosis [18] as primary causes of RDC. It is very challenging to differentiate between SIF and RDC by plain radiographs at any stage [8]. However, SIF of the femoral head was seen to progress rapidly to RDC in radiographic images. Furthermore, Niimi et al. have speculated that SIF is involved in the pathogenesis of RDC as a very important preliminary event [8].

In patients with SIF or RDC, joint pain occurs presumably because of femoral head collapse [3, 4, 8]. Our own case showed obvious joint space narrowing with hip joint pain prior to femoral head collapse. Although it is not fully understood why joint pain occurred only at an early stage, which is inconsistent with joint space narrowing and femoral head destruction, we speculate that pain might be halted when bone destruction stops. Finally, joint pain may disappear after the joint line shows osteosclerotic changes.

In this case, femoral head collapse progressed slowly over several years without obvious joint pain. Mashiba et al. reviewed that $\mathrm{BP}$ was effective for bone fracture healing, in which BP increased callus volume in immature bones [19].
Nishii et al. have suggested that alendronate has the potential to prevent collapse of the femoral head, even in patients with extensive necrosis, presumably by inhibiting bone resorption in the necrotic region [20]. These findings indicate that BP treatment might have prevented bone destruction and disease progression. BP may also have retarded the development of bone collapse and remarkably improved joint pain in our case. On the other hand, Chen et al. reported that alendronate had no effect on reducing OA progression or improving quality of life in their study [21].

This paper describes a patient initially diagnosed as having radiographic $\mathrm{OA}$, which then developed to possible $\mathrm{ON}$ and/or SIF, and finally appeared as RDC by plain radiographic examination. Burr et al. have reported that RDC of the femoral head also occurs in severely osteoporotic patients without any trauma but with acute joint pain [5]. SIF of the femoral head often afflicts patients with osteoporosis or osteopenia [3, 4]. In this study, bone destruction occurred slowly, probably because of BP treatment. We have recently uncovered that radiographic OA progresses rapidly in elderly people with accompanying bone alternations [22]. Therefore, RDC might result from bone destruction caused by bone fragility and rapid femoral head collapse may depend on the degree of bone fragility.

Previous reports have suggested that one of the pathophysiologies of OA is microfracture [23, 24]. Under this notion, the disorders of OA, SIF, ON, and RDC might share a similar pathophysiology. If the etiology of these conditions includes microfracture, disease status and progression may vary based on the degree of cartilage and/or bone destruction and stage in each disorder.

The main limitations of this report are 1) no axial view plain radiographs and 2) no MRI examinations of the affected right hip joint. Further studies will be required to better understand the pathophysiology of OA, ON, SIF, and RDC.

In conclusion, we encountered an elderly patient with an atypical clinical course that was radiographically diagnosed as OA, ON, SIF, and/or RDC over long-term follow-up. Diagnosis was challenging due to radiographic overlap and atypical disease progression. Thus, radiographic diagnosis appears to be affected by the timing of imaging evaluation and may benefit from complementary imaging modalities, such as MRI.

\section{CONFLICT OF INTEREST}

The authors confirm that this article content has no conflict of interest.

\section{ACKNOWLEDGEMENTS}

Declared none.

\section{REFERENCES}

[1] Sulsky SI, Carlton L, Bochmann F, et al. Epidemiological evidence for work load as a risk factor for osteoarthritis of the hip: a systematic review. PLoS One 2012; 7: e31521.

[2] Assouline-Dayan Y, Chang C, Greenspan A, Shoenfeld Y, Gershwin ME. Pathogenesis and natural history of osteonecrosis. Semin Arthritis Rheum 2002; 32: 94-124. 
[3] Yamamoto T. Subchondral insufficiency fractures of the femoral head. Clin Orthop Surg 2012; 4: 173-80.

[4] Godefroy D, Rousselin B, Sarazin L. Hip degeneration. J Radiol 2011; 92: 581-93. (Article in French).

[5] Burr DB, Gallant MA. Bone remodelling in osteoarthritis. Nat Rev Rheumatol 2012; 8: 665-73.

[6] Sugano N, Atsumi T, Ohzono K, Kubo T, Hotokebuchi T, Takaoka $\mathrm{K}$. The 2001 revised criteria for diagnosis, classification, and staging of idiopathic osteonecrosis of the femoral head. J Orthop Sci 2002; 7: 601-5.

[7] Iwasaki K, Yamamoto T, Motomura G, Mawatari T, Nakashima Y, Iwamoto Y. Subchondral insufficiency fracture of the femoral head in young adults. Clin Imaging 2011; 35: 208-13.

[8] Niimi R, Hasegawa M, Sudo A, Uchida A. Rapidly destructive coxopathy after subchondral insufficiency fracture of the femoral head. Arch Orthop Trauma Surg 2005; 125: 410-3.

[9] Heijink A, Gomoll AH, Madry $\mathrm{H}$, et al. Biomechanical considerations in the pathogenesis of osteoarthritis of the knee. Knee Surg Sports Traumatol Arthrosc 2012; 20: 423-35.

[10] Saini A, Saifuddin A. MRI of osteonecrosis. Clin Radiol 2004; 59: 1079-93. (Review).

[11] Ikemura S, Yamamoto T, Motomura G, Nakashima Y, Mawatari T, Iwamoto Y. MRI evaluation of collapsed femoral heads in patients 60 years old or older: differentiation of subchondral insufficiency fracture from osteonecrosis of the femoral head. AJR Am J Roentgenol 2010; 195: W63-8.

[12] Kellgren JH, Lawrence JS. Radiological assessment of osteoarthrosis. Ann Rheum Dis 1957; 16: 494-502.

[13] Kamimura M, Nakamura Y, Ikegami S, UchiyamaS, Kato H. Joint pain undergoes a transition in accordance with signal changes of bones detected by MRI in hip osteoarthritis. Open Rheumatol J 2013; 7: 58-65.

[14] Anjan P Kaushik, Anusuya Das, Quanjun Cui. Osteonecrosis of the femoral head: an update in year 2012. World J Orthop 2012; 3: 4957.
[15] Watanabe W, Sato K, Itoi E, Yang K, Watanabe H. Posterior pelvic tilt in patients with decreased lumbar lordosis decreases acetabular femoral head covering. Orthopedics 2002; 25: 321-4.

[16] Ryu KN, Kim EJ, Yoo MC, Park YK, Sartoris DJ, Resnick D. Ischemic necrosis of the entire femoral head and rapidly destructive hip disease: potential causative relationship. Skeletal Radiol 1997; 26: 143-9.

[17] Doherty M, Holt M, MacMillan P, Watt I, Dieppe P. A reappraisa of 'analgesic hip.' Ann Rheum Dis 1986; 45: 272-6.

[18] Menkes CJ, Simon F, Delrieu F, Forest M, Delbarre F. Destructive arthropathy in chondrocalcinosis articularis. Arthritis Rheum 1976; 19(Suppl 3): 329-48

[19] Mashiba T, Mori S. Bone fracture and the healing mechanisms The effects of anti-resorptive agents on fracture healing. Clin Calcium 2009; 19: 673-9.

[20] Nishii T, Sugano N, Miki H, Hashimoto J, Yoshikawa H. Does alendronate prevent collapse in osteonecrosis of the femoral head? Clin Orthop Relat Res 2006; 443: 273-9.

[21] Chen CH, Chang JK, Lai KA, Hou SM, Chang CH, Wang GJ. Alendronate in the prevention of collapse of the femoral head in nontraumatic osteonecrosis: a two-year multicenter, prospective, randomized, double-blind, placebo-controlled study. Arthritis Rheum 2012; 64: 1572-8.

[22] Kamimura M, Nakamura Y, Ikegami S, et al. The pathophysiology of primary hip osteoarthritis may originate from bone alterations. Open Rheumatol J 2013; 7: 112-8.

[23] Taljanovic MS, Graham AR, Benjamin JB, et al. Bone marrow edema pattern in advanced hip osteoarthritis: quantitative assessment with magnetic resonance imaging and correlation with clinical examination, radiographic findings, and histopathology. Skeletal Radiol 2008; 37: 423-31.

[24] Guermazi A, Roemer FW, Haugen IK, Crema MD, Hayashi D MRI-based semiquantitative scoring of joint pathology in osteoarthritis. Nat Rev Rheumatol 2013; 9: 236-51.

(C) Nakamura et al.; Licensee Bentham Open.

This is an open access article licensed under the terms of the Creative Commons Attribution Non-Commercial License (http://creativecommons.org/licenses/by-nc/ 3.0/) which permits unrestricted, non-commercial use, distribution and reproduction in any medium, provided the work is properly cited. 